OPEN ACCESS

Edited by:

Yingli Yang,

UCLA Health System, United States

Reviewed by:

Weiwei Zong,

Henry Ford Health System,

United States

Feng Liu,

Avera McKennan Hospital and

University Health Center,

United States

*Correspondence:

Bao-Tian Huang

hbt830910@126.com

${ }^{\dagger}$ These authors have contributed equally to this work and share first authorship

Specialty section: This article was submitted to Radiation Oncology, a section of the journal

Frontiers in Oncology

Received: 20 November 2021

Accepted: 31 December 2021

Published: 31 January 2022

Citation:

Luo L-M, Huang B-T, Chen C-Z,

Wang $Y$, Su $C-H$, Peng $G-B$,

Zeng $C-B$, Wu $Y-X$, Wang $R-H$,

Huang K and Qiu Z-H (2022) A

Combined Model to Improve the

Prediction of Local Control for Lung

Cancer Patients Undergoing

Stereotactic Body Radiotherapy Based on Radiomic Signature Plus Clinical and Dosimetric Parameters.

Front. Oncol. 11:819047.

doi: 10.3389/fonc.2021.819047

\section{A Combined Model to Improve the Prediction of Local Control for Lung Cancer Patients Undergoing Stereotactic Body Radiotherapy Based on Radiomic Signature Plus Clinical and Dosimetric Parameters}

\author{
Li-Mei Luo ${ }^{1,2 \dagger}$, Bao-Tian Huang ${ }^{2 \star t}$, Chuang-Zhen Chen ${ }^{2}$, Ying Wang ${ }^{1,2}$, \\ Chuang-Huang $\mathrm{Su}^{3}$, Guo-Bo Peng ${ }^{4}$, Cheng-Bing Zeng ${ }^{2}$, Yan-Xuan $\mathrm{Wu}^{2}$, \\ Ruo-Heng Wang ${ }^{1,2}$, Kang Huang ${ }^{1,2}$ and Zi-Han Qiu ${ }^{5}$ \\ ${ }^{1}$ Department of Radiation Oncology, Shantou University Medical College, Shantou, China, ${ }^{2}$ Department of Radiation \\ Oncology, Cancer Hospital of Shantou University Medical College, Shantou, China, ${ }^{3}$ Department of Radiation Oncology, \\ Shantou Central Hospital, Shantou, China, ${ }^{4}$ Department of Radiation Oncology, Meizhou People's Hospital (Huangtang \\ Hospital), Meizhou Academy of Medical Sciences, Meizhou, China, ${ }^{5}$ Department of Otolaryngology-Head and Neck Surgery, \\ The First Affiliated Hospital of Shantou University Medical College, Shantou, China
}

Purpose: Stereotactic body radiotherapy (SBRT) is an important treatment modality for lung cancer patients, however, tumor local recurrence rate remains some challenge and there is no reliable prediction tool. This study aims to develop a prediction model of local control for lung cancer patients undergoing SBRT based on radiomics signature combining with clinical and dosimetric parameters.

Methods: The radiomics model, clinical model and combined model were developed by radiomics features, incorporating clinical and dosimetric parameters and radiomics signatures plus clinical and dosimetric parameters, respectively. Three models were established by logistic regression (LR), decision tree (DT) or support vector machine (SVM). The performance of models was assessed by receiver operating characteristic curve $(\mathrm{ROC})$ and DeLong test. Furthermore, a nomogram was built and was assessed by calibration curve, Hosmer-Lemeshow and decision curve.

Results: The LR method was selected for model establishment. The radiomics model, clinical model and combined model showed favorite performance and calibration (Area under the ROC curve (AUC) 0.811, 0.845 and 0.911 in the training group, 0.702, 0.786 and 0.818 in the validation group, respectively). The performance of combined model was significantly superior than the other two models. In addition, Calibration curve and Hosmer-Lemeshow (training group: $P=0.898$, validation group: $P=0.891$ ) showed good calibration of combined nomogram and decision curve proved its clinical utility. 


\section{Conclusions: The combined model based on radiomics features plus clinical and dosimetric parameters can improve the prediction of 1-year local control for lung cancer patients undergoing SBRT.}

Keywords: lung cancer, stereotactic body radiotherapy, local control, radiomics, clinical, dosimetric, prediction model

\section{INTRODUCTION}

Lung cancer is the second most common cancer and the main cause of cancer-related deaths, more than 2.21 million patients worldwide are affected every year (1). With the improvement of radiotherapy technology, stereotactic body radiation therapy (SBRT) is generally recognized as a standard option for early stage lung cancer patients who are not fit or healthy enough to be candidates for surgery or who refuse operation due to various complications (2). SBRT is also well established in the treatment of oligometastatic patients, e.g., with pulmonary metastases (3). This precise modality uses high doses to ablative cancer target with low doses to protect surrounding tissue. The local control rate in 5 years after SBRT is about $72 \%$ with a median follow-up of 4 years for early stage localized tumors (4). Furthermore, a variety of studies have reported local control is excellent after SBRT; however, there are still patients suffering from local recurrence (5). Therefore, a model for accurately and individually predicting the local control status for lung cancer patients after SBRT is highly desirable.

The maximum standardized uptake value (SUVmax) in PETCT was used to predict local recurrence after SBRT, but the results varied from institutions to patient groups, suggesting that its prognostic value was uncertain $(6,7)$. Several studies reported some clinical and dosimetric factors were influential parameters for local control prediction (8-11) and dose-response model to calculate local control possibility for lung SBRT patients employed clinical and dosimetric parameters were established (12-15). However, their models did not accurately predict patients' outcome, while other tumor individual characteristics were not considered. A comprehensive and noninvasive approach based on individual heterogeneous to screen candidate patients with tumor local control status is necessary.

Radiomics is based on the extraction of tumor features from traditional medical images to predict treatment effectiveness and prognosis of different diseases, including lung cancer, esophageal cancer, and prostate cancer (16-19). Moreover, radiomics has prognostic value in predicting clinical outcomes of pulmonary SBRT $(20,21)$. However, few prediction models considering radiomic signature combined with clinical and dosimetric parameters has been proposed to evaluate the tumor local control for lung cancer patients undergoing SBRT.

Therefore, the aim of our study is to generate a robust combined model for predicting 1-year tumor local control in primary and secondary lung cancer patients treated with SBRT by integrating radiomic signature and clinical and dosimetric parameters.

\section{MATERIALS AND METHODS}

The workflow of the study is shown in Figure 1.

\section{Patients' Population and Treatment}

We firstly analyzed retrospectively registered data from July 27, 2011 to December 7, 2018 of patients diagnosed with primary and secondary lung cancer and treated with lung SBRT in Cancer Hospital of Shantou $(N=134)$. Next, some patients with irradiation sites including chest wall, mediastinum, and thoracic vertebra $(N=4)$, who were lost to follow-up $(N=18)$ and did not complete the treatment course $(N=1)$ were excluded from the analysis. Finally, 119 patients with 18 patients had repeat lesions and 129 tumors were available for next analysis. The study was conducted in accordance with the Declaration of Helsinki and approved by the ethical board; however, patient written informed consent was waived. Our patients were staged by using the seventh editions of the AJCC staging system. Tumors were simulated via four-dimensional computed tomography (4DCT) or three-dimensional computed tomography (3DCT). The internal target volume (ITV) with 4DCT was defined by combining gross tumor volumes (GTVs) contoured at 10 respiratory phases. In addition, some ITV with 3DCT was defined by two GTVs contoured at the peak-exhale and peak-inhale respiratory phases; other ITV with 3DCT was defined by observing the tumor motion amplitude obtained from fluoroscopy. The planning target volume (PTV) was delineated by adding $5 \mathrm{~mm}$ of ITV in all directions. Cone beam computed tomography (CBCT) was used for image guidance and tumor localization before each fraction with correction. All patients were randomly assigned to the training group and validation group in a ratio of $7: 3$.

\section{Follow-Up}

Patients were evaluated with CT scans repeated every 3 months after the treatment in the first year, and then every 6 months thereafter (4). Tumor local control is defined as the absence of local recurrence of the tumor at the treatment site. Local recurrence was defined by pathologic confirmation, mass progression of the primary tumor, and the involved lobe on two consecutive CT at least 6 months, or the discretion of oncologists based on clinical symptoms and signs of patients $(14,22)$.

\section{Clinical and Dosimetric Parameters Data Collection}

We collected patients' baseline clinical and dosimetric parameters and 1-year tumor local status after SBRT. All the 


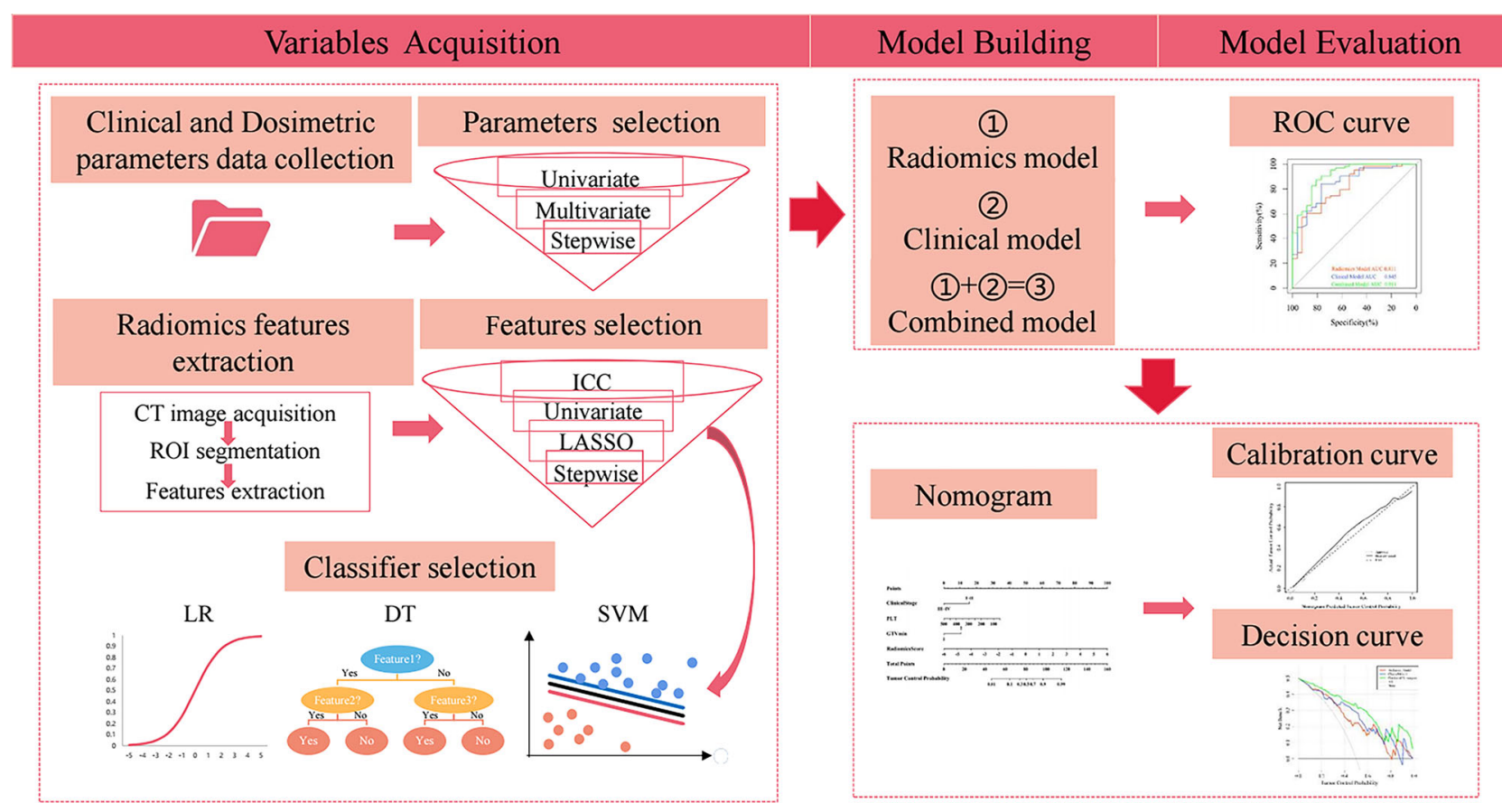

FIGURE 1 | Workflow of the study. ROI, region of interest; ICC, intraclass correlation coefficient; LASSO, least absolute shrinkage and selection operator; LR, logistic regression; DT, decision tree; SVM, support vector machine. ROC, receiver operating characteristic.

doses mentioned in this paper were biologically effective doses (BEDs), the linear-quadratic model with an $\alpha / \beta$ ratio of $10 \mathrm{~Gy}$ was adopted for calculating BEDs, BED $=n \times d \times[1+d /(\alpha / \beta)]$, where $n$ is the fraction number and $d$ is the fractional dose (15). Clinical data included gender, age, smoking status, Karnofsky performance status (KPS), body mass index (BMI), clinical stage, location, histology, equivalent diameter, GTV, PTV, chemotherapy or not, lymphocyte, neutrophil, platelet (PLT), neutrophil-to-lymphocyte ratio (NLR), platelet-to-lymphocyte ratio (PLR), hemoglobin ( $\mathrm{Hb})$, tumor site, immobilization device, and 4DCT or not. Dosimetric data included the prescription dose that covers $95 \%$ of the target area expressed as $\operatorname{BED}\left(D_{95}\right)$ and the maximum dose in the whole plan $\left(D_{\max }\right)$; the minimum dose of PTV $\left(\mathrm{PTV}_{\min }\right)$, mean dose of PTV $\left(\mathrm{PTV}_{\text {mean }}\right)$, and maximum dose of PTV $\left(\mathrm{PTV}_{\max }\right)$ and dose inhomogeneity in PTV $\left(\mathrm{PTV}_{\min } / \mathrm{PTV}_{\max }\right)$; and the minimum dose of GTV $\left(\mathrm{GTV}_{\min }\right)$, mean dose of GTV $\left(\mathrm{GTV}_{\text {mean }}\right)$, and the maximum dose of GTV $\left(\mathrm{GTV}_{\max }\right)$ and dose inhomogeneity in GTV $\left(\mathrm{GTV}_{\min } / \mathrm{GTV}_{\max }\right)$.

\section{Clinical and Dosimetric Parameter Selection}

The univariate logistic regression (LR) analysis was applied to evaluate whether parameters were candidate predictors of 1-year tumor local control and a stepwise multivariate LR was used to determine the best variables of statistically significant parameters in univariate analysis.

\section{CT Image Acquisition, Region of Interest Segmentation and Quantitative Radiomics Features Extraction}

All patients underwent CT scanning prior to SBRT treatment using a Brilliance Big Bore CT (Philips Brilliance CT Big Bore Oncology Configuration, Cleveland, OH, USA). The detailed information of the CT scanners was as follows: tube voltage of $120 \mathrm{kVp}$, tube current of $350 \mathrm{~mA}$, convolution kernel of standard, and construction matrix of $512 \times 512$. The scanning range was from the apex to the bottom of the lung. CT images were then transferred to an Eclipse treatment planning system (Version 10.0, Varian Medical System, Inc., Palo Alto, CA, USA) for the whole tumor delineation, also known as the region of interest (ROI) segmentation by one radiology doctor with more than 10 years of work experience (23).

Radiomics features were automatically extracted from each tumor segmentation using PyRadiomics (https://github.com/ Radiomics/pyradiomics). The images which were used to extract the radiomics features could be either the original image or the derivative filtered images including Laplacian of Gaussian (LoG), Wavelet, Square, SquareRoot, Logarithm. Collectively, the feature types extracted from each image type include shape features provided the geometric volume of ROI, first-order features described the individual voxel value distribution in the intensity histogram of ROI, texture features reflected the organization and arrangement of the surface structure with slow change or periodic change, including gray- 
level co-occurrence matrix (GLCM), gray-level dependence matrix (GLDM), gray-level run length matrix (GLRLM), graylevel size zone matrix (GLSZM), and neighborhood gray-tone difference matrix (NGTDM) (24). In order to ensure the repeatability of the results, the images and features were resampled and z-score normalized respectively.

\section{Radiomics Feature Selection}

A large number of radiomic features may result in overfitting of the model, reducing the predictive performance of the model. To overcome the dimensional disaster and reduce the bias caused by many radiomics features, we gradually use four methods of the intraclass correlation coefficient (ICC), univariant analysis, least absolute shrinkage and selection operator (LASSO), and stepwise regression to select the vital features from the training group. First, in order to minimize the differences between observers and enhance the robustness of features, two radiology doctors independently delineated 30 randomly chosen samples drawn from patients, and then the ICC was calculated from the extracted features of these 30 cases to assess intraobserver and interobserver reproducibility. The features with ICC $>0.75$ were considered stable for the further analysis. The ICC was conducted by using the "irr" package in R software (25). Subsequently, we evaluated remaining radiomics features using the independent samples $t$-test or the Mann-Whitney $U$ test to collect statistically significant features with a $p$-value of $<0.05$. In addition, to deal with the high-dimensional data and enhance the prediction accuracy, the LASSO regression, as an effective dimensionality reduction method, was applied to select potentially important features by regularizing concurrently. The optimal area under curve and parameter $\log (\lambda)$ were determined through 10 -fold cross-validation to control the complexity of the model and select the most robust and nonredundant radiomics features $(26,27)$. The LASSO logistic regression was conducted by using the "glmnet" package (28). Finally, stepwise regression is used to eliminate the redundant features to avoid the multicollinearity. Moreover, the importance of the most valuable features were analyzed and evaluated by correlation analysis.

\section{Classifier Selection}

We fed the final selected radiomics features into the classifiers to build the optimal radiomics model. In our study, LR, decision tree (DT), and support vector machine (SVM) were used to build and evaluate radiomics model, and the best classification method was selected for subsequent analysis (29-31). The DT model was performed using the "rpart" package (32), while the "e1071" package was employed to develop the SVM model (28), all of them are carried out by tuning the parameters. To complement the analyses, the radiomics signature (radiomics score) was calculated using the radiomics features. The best classifier was adopted for building clinical model and combined model.

\section{Prediction Model Development}

Accordingly, three different prediction models were described briefly as: the radiomics model composed of radiomics signature, the clinical model constructed from clinical and dosimetric parameters, and the combined model developed by combining radiomics signature and clinical and dosimetric parameters.

\section{Model Performance Comparison}

Based on receiver operating characteristic (ROC) curve, the prediction models were compared by calculating the area under the ROC curve (AUC) values, $p$-value, accuracy, sensitivity, specificity, and DeLong test. The ROC curves were plotted based on the "pROC" package (33).

\section{Nomogram Construction and Validation}

In order to visually and individually predict the tumor control probability (TCP) of lung cancer after SBRT, we created a nomogram which was developed by the prediction model with the best performance in the training group. The ability of the nomogram was conducted by the calibration curve and the Hosmer-Lemeshow test. The net benefits and the clinical usefulness of three models for prognosis was measured and compared by the decision curve analysis. The nomogram and the DCA were plotted using the "rms" package and the "dca.R." package, respectively (33).

\section{Statistical Analysis}

Statistical analyses were performed based on SPSS v.23.0 (SPSS Inc., Chicago, IL, USA) and R software v.4.0.2 (R Project for Statistical Computing, Vienna, Austria). The Student's $t$-test or Mann-Whitney $U$ test was employed to compare continuous variables, and the Chi-square test or Fisher's exact test was applied for categorical variables. The optimal cutoff point was assessed by using the Youden's index on the ROC curve (34). An AUC comparison of the three prediction models with the best classifier methods was performed by DeLong test. The tests were two-sided, and $p$-values less than 0.05 were considered statistically significant.

\section{RESULTS}

\section{Clinical and Dosimetric Parameters of Patients}

Data for 129 tumors from 111 primary and secondary lung cancer patients treated with SBRT were available, of which 89 and 40 tumors were divided into the training and validation group, respectively. Baseline characteristics are presented on Table S1. Males constituted $93(72.1 \%)$ of the sample. Mean age was 62 years. Most tumors (82.9\%) were at a peripheral location compared with other location. A minority of tumors (18.6\%) were treated with combined radiotherapy and concurrent chemotherapy. The median prescription dose was 48 Gy (range 18-70) delivered in a median of 4 fractions (range $1-12$ ), and the median prescription dose in $\mathrm{BED}_{95}$ was $95.2 \mathrm{~Gy}$ (range 28.8-180). The distribution of fractionation schemes and $\mathrm{BED}_{95}$ used in our study was described in Figure 2; most used dose-fractionation scheme was $50 \mathrm{~Gy}$ in 4 fractions. One year after SBRT treatment, 91 tumors were local controlled and 38 local failures were observed. The optimal cutoff values of 


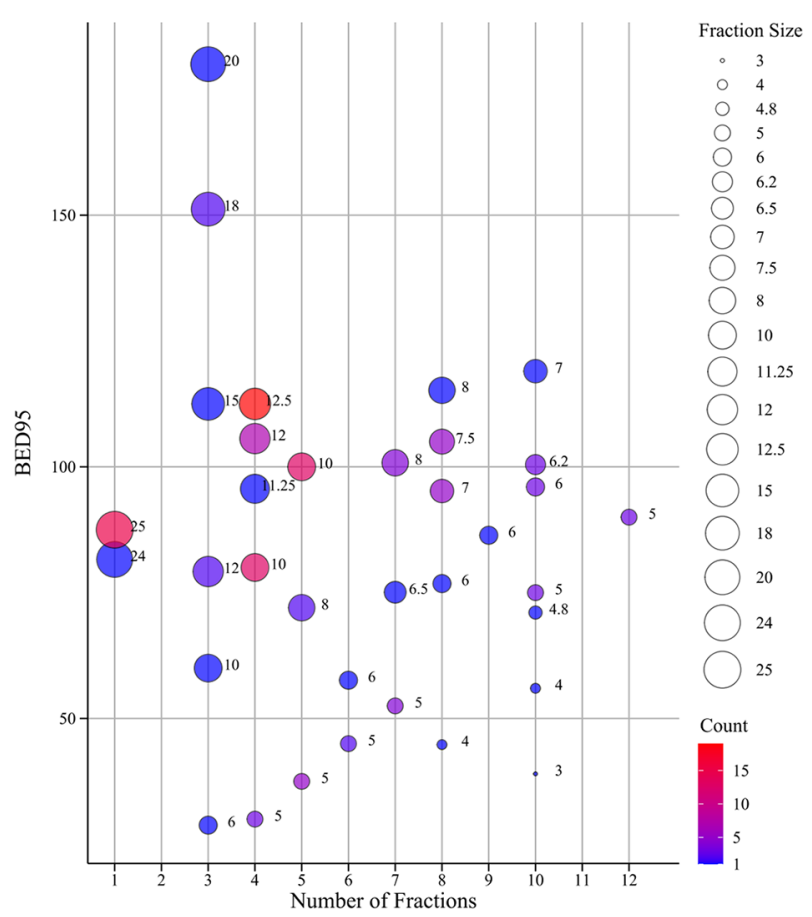

FIGURE 2 | The bubble chart of fractionation schemes and BED 95 . The size of dots indicates the size of fraction; the different color of dots indicates different count ranges. $\mathrm{BED}_{95}$, the prescription dose covers $95 \%$ of the target area expressed as BED.

dosimetric parameters based on ROC curve are shown in Table 1. No significant association was seen with baseline clinical and dosimetric characteristics in the training group and validation group of tumors. The balance of the two sets of data suggests that the patient grouping was reasonable.

\section{Clinical and Dosimetric Parameter Selection}

The relationship between clinical and dosimetric parameters and the 1-year tumor local status of primary and secondary lung cancer after SBRT for the training group is summarized in Table 2. In the univariate analysis, clinical stage status, history, PLT, PLR, Hb, $\mathrm{D}_{95}, \mathrm{D}_{\max }, \mathrm{PTV}_{\text {min }}, \mathrm{PTV}_{\max }, \mathrm{PTV}_{\text {mean }}, \mathrm{GTV}_{\text {min }}$, $\mathrm{GTV}_{\text {max }}, \mathrm{GTV}_{\text {mean }}$, and $\mathrm{PTV}_{\min } / \mathrm{PTV}_{\max }$ were found to be significantly different between the 1 -year local control status and the local failure status (all $p<0.05$ ). Multivariate analysis indicated that clinical stage status, platelet (PLT), and the minimum dose of gross tumor volume $\left(\mathrm{BEDGTV}_{\min }\right)$ were prognostic parameters for 1-year tumor local status.

\section{Radiomics Feature Selection}

Radiomics features were extracted and selected using the procedure shown in Figure 3. In total, 1,502 radiomics features were successfully extracted from each three-dimensional ROI, including 14 shape features, 288 first-order features, and 1,200 texture features. For intraobserver agreement, 1,090 features with ICC $\geqq 0.75$ between observers were included in further analyses. According to the univariate analysis, 46 radiomics features were collected, and then 10 potential radiomics features were calculated by the LASSO regression model with a penalty parameter $\lambda=0.025$; we finally performed the stepwise regression analysis and obtained 4 important radiomics features, namely wavelet-LLL_glszm_SmallAreaEmphasis, wavelet-LHH_glcm_Joint Average, wavelet-LHH_ngtdm Complexity, and squareroot_glcm_DifferenceEntropy. In the training group, the visible distributions of these radiomics features in local control group and local failure group and the correlation analysis of radiomics features are shown in Figures 4, 5. It indicated that the larger the value of each radiomics features, the greater the possibility of 1-year tumor local control

TABLE 1 | The optimal cut-off values of dosimetric parameters.

\begin{tabular}{lcccccccc}
\hline Dosimetric parameters & BED $_{\mathbf{9 5}}$ & BED $_{\text {max }}$ & BEDPTV $_{\text {min }}$ & BEDPTV $_{\text {max }}$ & BEDPTV $_{\text {mean }}$ & BEDGTV $_{\text {min }}$ & BEDGTV $_{\text {max }}$ & BEDGTV $_{\text {mean }}$ \\
\hline Cutoff values & 84.00 & 110.85 & 80.43 & 110.85 & 101.73 & 98.79 & 103.87 \\
\hline
\end{tabular}

$B E D_{95}$, the prescription dose covers $95 \%$ of the target area expressed as BED; BED max the maximum dose in the whole plan; BEDPTV $V_{\text {min }}$, the minimum dose of PTV; BEDPTV mean, mean dose of PTV; BEDPTV max, the maximum dose of PTV; BEDGTV min, the minimum dose of GTV; BEDGTV mean, mean dose of GTV; BEDGTV max, the maximum dose of GTV. 
TABLE 2 | Univariate and multivariate analyses of clinical and dosimetric parameters.

\begin{tabular}{|c|c|c|c|c|c|c|}
\hline \multirow[t]{2}{*}{ Variable } & \multicolumn{2}{|c|}{ Univariate analyses (logistic) } & \multicolumn{2}{|c|}{ Multivariate analyses (logistic) } & \multicolumn{2}{|c|}{ Multivariate analyses (stepwise) } \\
\hline & $p$-value & $\beta$ & $p$-value & $\beta$ & $p$-value & $\beta$ \\
\hline Sex (man vs. woman) & 0.299 & -0.527 & & & & \\
\hline Age (years) & 0.615 & -0.010 & & & & \\
\hline \multicolumn{7}{|l|}{ Smoking status } \\
\hline Current & Reference & Reference & & & & \\
\hline Former & 0.990 & -17.806 & & & & \\
\hline Never & 0.302 & -0.506 & & & & \\
\hline KPS (<80 vs. $\geq 80)$ & 0.080 & 1.256 & & & & \\
\hline $\mathrm{BMI}\left(\mathrm{kg} / \mathrm{m}^{2}\right)$ & 0.914 & 0.008 & & & & \\
\hline Clinical stage (I II vs. III IV) & 0.001 & -2.132 & 0.214 & -1.284 & 0.034 & -1.543 \\
\hline Location (central vs. peripheral) & 0.252 & -0.784 & & & & \\
\hline \multicolumn{7}{|l|}{ Histology } \\
\hline Adenocarcinoma & Reference & Reference & Reference & Reference & & \\
\hline Squamous cell carcinoma & 0.405 & -0.597 & 0.733 & -0.425 & & \\
\hline Unknown & 0.020 & -1.477 & 0.931 & 0.101 & & \\
\hline Equivalent diameter (cm) & 0.197 & -0.177 & & & & \\
\hline $\operatorname{GTV}\left(\mathrm{cm}^{2}\right)$ & 0.184 & -0.005 & & & & \\
\hline $\operatorname{PTV}\left(\mathrm{cm}^{2}\right)$ & 0.140 & -0.004 & & & & \\
\hline Chemotherapy (yes vs. no) & 0.431 & 0.493 & & & & \\
\hline Lymphocyte $\left(10^{9} / \mathrm{L}\right)$ & 0.056 & 0.698 & & & & \\
\hline Neutrophil $\left(10^{9} / \mathrm{L}\right)$ & 0.176 & -0.134 & & & & \\
\hline $\operatorname{PLT}\left(10^{9} / \mathrm{L}\right)$ & 0.006 & -0.010 & 0.115 & -0.009 & 0.038 & -0.009 \\
\hline NLR & 0.128 & -0.135 & & & & \\
\hline PLR & 0.011 & -0.008 & 0.435 & -0.004 & & \\
\hline $\mathrm{Hb}(\mathrm{g} / \mathrm{L})$ & 0.031 & 0.037 & 0.376 & 0.021 & & \\
\hline Immobilization device (vacuum bag vs. thermoplastic mask) & 0.144 & 0.693 & & & & \\
\hline 4DCT (yes vs. no) & -0.851 & 0.109 & & & & \\
\hline BED $_{95}(<84.00$ vs. $\geq 84.00)$ (Gy) & 0.001 & 1.723 & 0.659 & 0.530 & & \\
\hline$B E D_{\max }(<110.85$ vs. $\geq 110.85)$ (Gy) & $<0.0001$ & 2.431 & 0.992 & 14.790 & & \\
\hline BEDPTV $_{\min }(<80.43$ vs. $\geq 80.43$ ) (Gy) & 0.004 & 1.484 & 0.396 & 1.386 & & \\
\hline BEDPTV $_{\max }(<110.85$ vs. $\geq 110.85)$ (Gy) & $<0.0001$ & 2.351 & 0.992 & -14.290 & & \\
\hline BEDPTV $_{\text {mean }}(<101.73$ vs. $\geq 101.73$ ) (Gy) & $<0.0001$ & 2.128 & 0.727 & 0.616 & & \\
\hline BEDGTV $_{\min }(<98.79$ vs. $\geq 98.79)(\mathrm{Gy})$ & $<0.0001$ & 2.258 & 0.510 & 0.848 & 0.009 & 1.699 \\
\hline BEDGTV $_{\max }(<103.87$ vs. $\geq 103.87)$ (Gy) & $<0.0001$ & 1.983 & 0.891 & -0.266 & & \\
\hline BEDGTV $_{\text {mean }}(<97.06$ vs. $\geq 97.06)$ (Gy) & $<0.0001$ & 1.864 & 0.433 & -1.752 & & \\
\hline$B E D P T V_{\min } / P T V_{\max }$ & 0.004 & -11.277 & 0.062 & -13.31 & 0.071 & -7.661 \\
\hline BEDGTV $\min / G_{T V}$ & 0.075 & -12.134 & & & & \\
\hline Tumor site (primary vs. secondary) & 0.398 & -0.399 & & & & \\
\hline
\end{tabular}

KPS, Karnofsky performance status; BMI, body mass index; PTV, planning target volume; GTV, gross tumor volume; PLT, platelet; Hb, hemoglobin; NLR, neutrophil-to-lymphocyte ratio; $P L R$, platelet-to-lymphocyte ratio; $4 D C T$, four-dimensional computed tomography; $B E D_{95}$, the prescription dose covers $95 \%$ of the target area expressed as $B E D$; $B E D_{\text {max }}$, the maximum dose in the whole plan; BEDPTV $V_{\text {min }}$, the minimum dose of PTV; BEDPTV mean, mean dose of PTV; BEDPTV max, the maximum dose of PTV; BEDPTV mir /PTV max, dose inhomogeneity in PTV; BEDGTV $_{\text {min }}$, the minimum dose of GTV; BEDGTV

and they were statistically supported. There was no significant correlation between the radiomics features with correlation coefficient $<0.75$.

\section{Classifier Selection}

The performance of model using difference classifiers are presented in Table 3. Our result reports that DT approach for classification were no more valuable than random classification with the AUC of the model below 0.5. We input these features into SVM classifier, which received the poor performance and the low specificity. Compared with the above methods, the LR was the optimum classifier with the best performance and accuracy.

\section{Prediction Model Construction}

On the basis of 4 radiomics factors, a radiomics model was created by the following formula:

$$
\begin{gathered}
\text { radiomics score }=-27.645+14.393 \\
\times \text { wavelet-LLL_glszm_SmallAreaEmphasis } \\
+8.075 \times \text { wavelet-LHH_glcm_JointAverage } \\
-3.386 \times \text { wavelet-LHH_ngtdm_Complexity } \\
+9.196 \times \text { squareroot_glcm_DifferenceEntropy }
\end{gathered}
$$

The formula and these coefficients were calculated from the LR. To illustrate the validity of the radiomics score at the nomogram, the visible distributions of radiomics score for the 1-year tumor local control and local failure groups in the training group and validation group are shown in Figure 4. With the quantitative value of score increased, the tumor can be more possibly locally controlled in a year. The parameters of clinical stage status, PLT, and BEDGTV $\mathrm{Bm}_{\text {min }}$ were employed to build the clinical model. Furthermore, the parameters plus radiomics score were brought into building the combined model. 


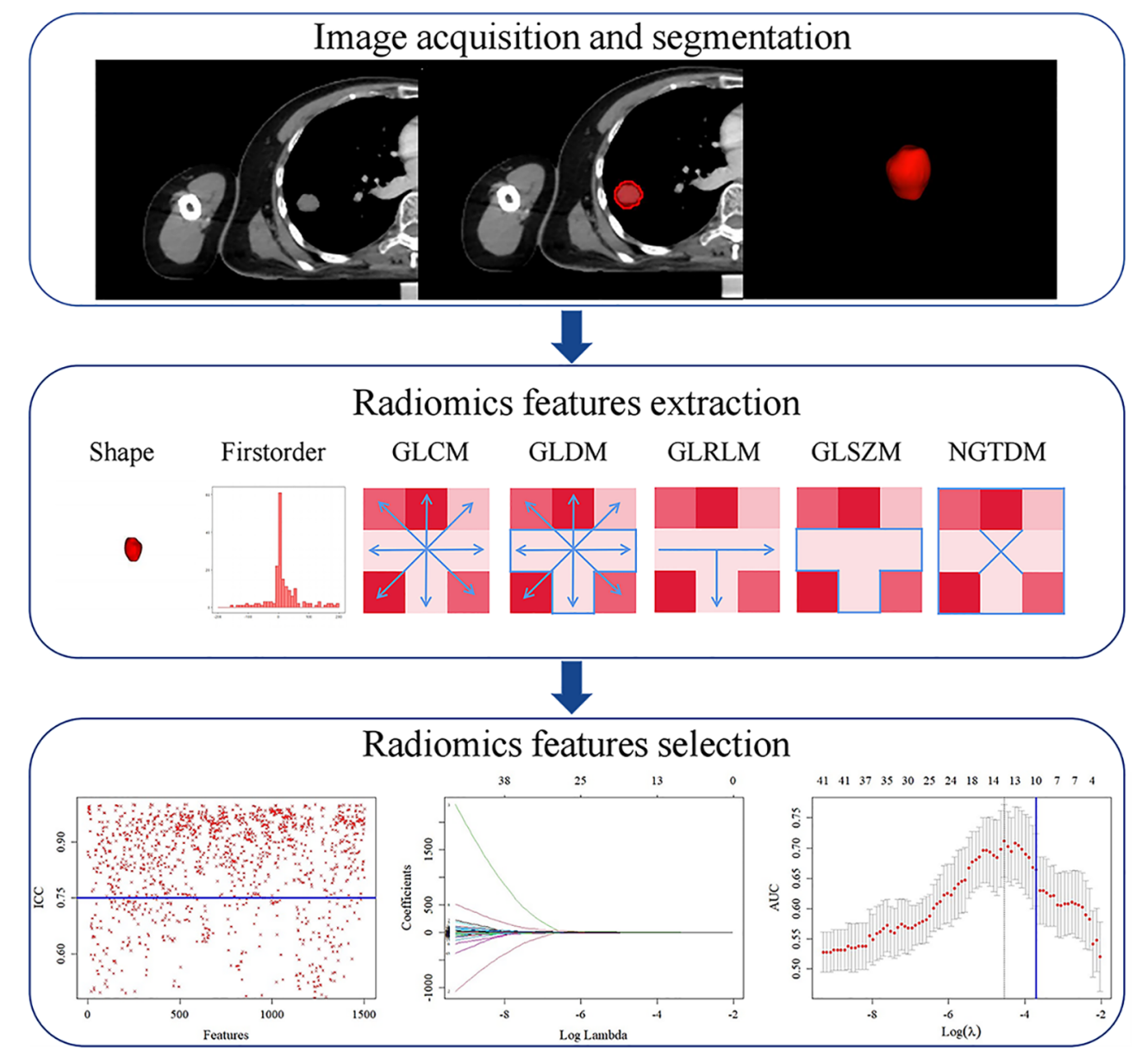

FIGURE 3 | Radiomics feature extraction and selection process. First, region of interest (ROI) segmentation was performed on CT image. Next, radiomics features were extracted from ROI. Finally, radiomics features dimension was reduced by intraclass correlation coefficient (ICC), least absolute shrinkage and selection operator (LASSO). GLCM, gray-level co-occurrence matrix; GLDM, gray-level dependence matrix; GLRLM, gray-level run length matrix; GLSZM, gray-level size zone matrix; NGTDM, neighborhood gray-tone difference matrix.

\section{Prediction Model Performance Comparison}

Figure 6 showed that the AUC with its 95\% confidence interval (CI) of the radiomics model, clinical model, and combined model were 0.811 (95\% CI: 0.713-0.910), 0.845 (95\% CI: $0.757-0.934)$, and 0.911 (95\% CI: $0.845-0.977)$ in the training group and 0.702 (95\% CI: 0.507-0.898), 0.786 (95\% CI: $0.638-$ 0.933 ), and 0.818 (95\% CI: $0.659-0.978$ ) in the validation group, respectively. The accuracy values of the radiomics model, clinical model, and combined model were $67.4 \%, 82.0 \%$ and $85.4 \%$ in the training group and $92.9 \%, 77.5 \%$, and $82.5 \%$ in the validation group, respectively. The combined model indicated a significant better performance than the radiomics model $(p=0.025)$ and the clinical model $(p=0.033)$ in the training group, while the radiomics model and clinical model displayed a similar performance $(p=0.613)$. We can also see the trend that the effect of the combined model is better than that of the single model in the validation group. Therefore, the optimal prediction model was based on a multivariable LR and conjoined the radiomics signature with clinical and dosimetric parameters. Moreover, the contribution of each selected feature in the combined model iss displayed in Figure 7.

\section{Nomogram Establishment}

A visualization-combined nomogram was constructed from integrating the radiomics score, clinical stage, PLT, and BEDGTV $_{\text {min }}$, as shown in Figure 8A. No significance was found in the Hosmer-Lemeshow test for the separated training sets $(p=0.898)$ and validation group $(p=0.891)$, indicating the proposed nomogram with good calibration was acceptable. The actual tumor control probability is that the patient population was divided into a few bins of increasing percentage of local control. The calibration curve of the combined nomogram confirmed that the probability of predicting 1-year tumor local control was consistent with the actual observation both in the training group (Figure 8B) and validation group (Figure $\mathbf{8 C}$ ). 
A

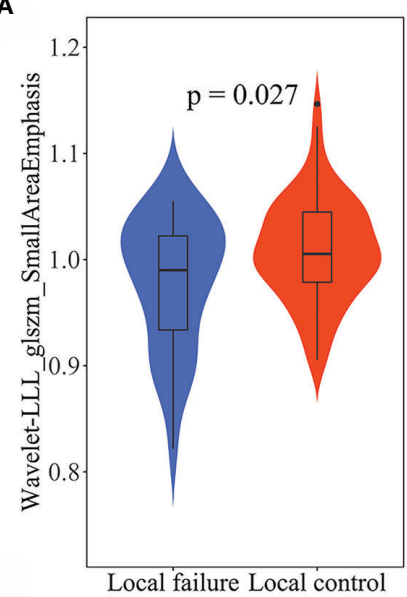

C

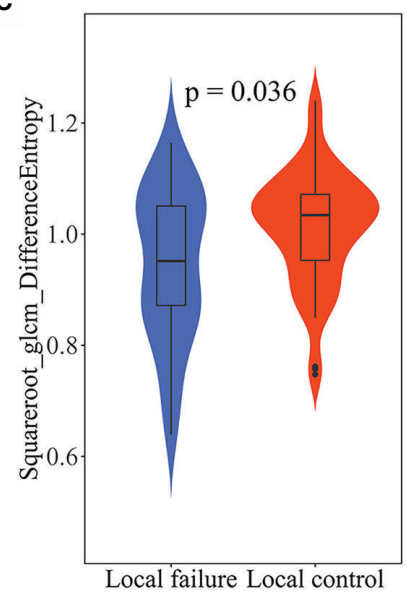

B

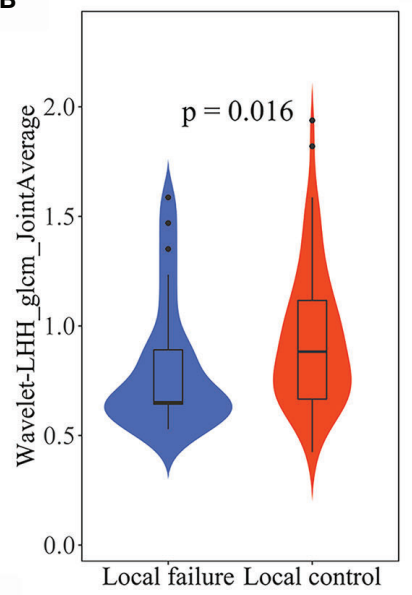

D

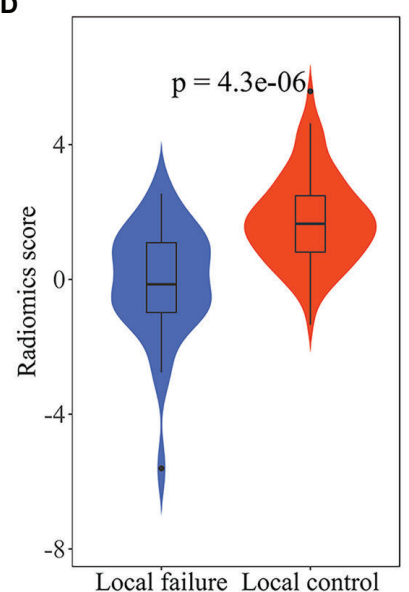

C

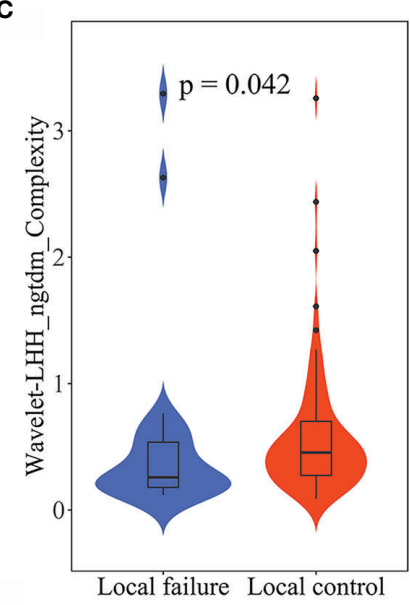

E

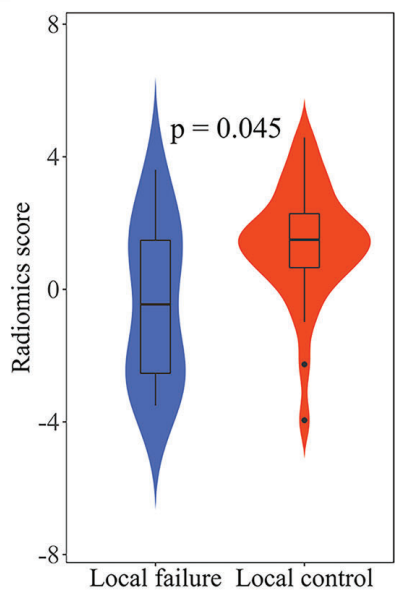

FIGURE 4 | The violin plots of radiomics features and radiomics score. The distribution of (A) wavelet-LLL_glszm_SmallAreaEmphasis, (B) wavelet-LHH_glcm_ JointAverage, (C) wavelet-LHH_ngtdm_Complexity, and (D) squareroot_glcm_DifferenceEntropy in the training group. The distribution of radiomics score in the training group (E) and in the validation group (F). The $p$-values were obtained by $t$-test or Wilcoxon rank sum test.

The decision curve revealed the radiomics model, the clinical model, and the combined nomogram were beneficial for predicting 1-year tumor local control probability. The area under the curve of the combined nomogram was larger than that of the other two models, indicating that the combined nomogram had the highest clinical feasibility and utility (Figures 8D, E).

\section{DISCUSSION}

In this study, a quantitative relationship between radiomics score, clinical and dosimetric features, and tumor local status was found. Moreover, we first established a new prediction model to correlate 1-year local control with radiomics score and clinical and dosimetric parameters for primary and secondary lung cancer patients undergoing SBRT. We also constructed and validated a combined nomogram with great discrimination to conveniently identify the tumor local status.
Improving the accuracy of local control prediction is of positive significance for medical and personal decision-making in many aspects (2). It is beneficial to find patients who are at high risk of locoregional failure and explore the treatment strategy of patients. Moreover, increasing systemic treatment and/or radiation dose to eradicate lesions and strengthening the follow-up management of patients can reduce local recurrence and improve the survival and prognosis. In clinical practice, some studies have proposed to overcome immune resistance mechanisms for lung cancer by using immunotherapy combined with SBRT; therefore, patients have more personalized treatment options $(35,36)$. Our nomogram model may provide important evidence to design future clinical trials, such as predicting whether these people would benefit from combination treatment to balance this positive strategic risk. More importantly, compared with the long-term outcome of overall survival, local control status avoids long-term follow-up and can early adjust treatment strategies. Therefore, our study provides a more effective tool to promptly achieve personalized treatment. 


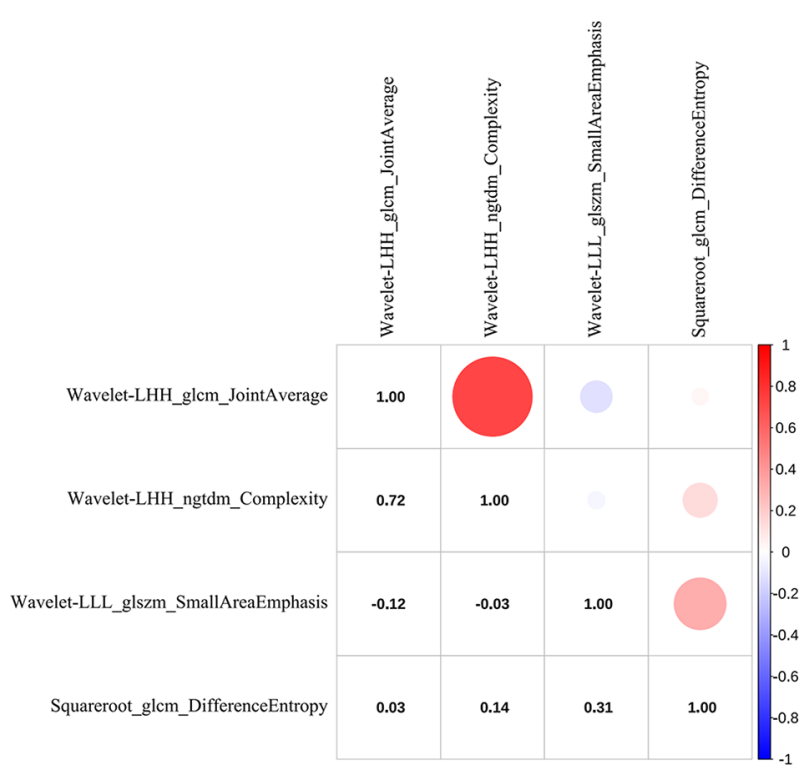

FIGURE 5 | The correlation heat map of radiomics features. Red indicates positive correlation, and blue indicates negative correlation; the darker the color, the stronger the relationship.

TABLE 3 | The performance of radiomics model using LR, DT, and SVM, clinical model and combined model.

\begin{tabular}{|c|c|c|c|c|c|c|}
\hline Group & Methods & AUC $(95 \% \mathrm{Cl})$ & $p$-value & Accuracy (\%) & Sensitivity (\%) & Specificity (\%) \\
\hline \multirow[t]{5}{*}{ Training } & LR & $0.811(0.713-0.910)$ & 0.000 & 67.4 & 57.1 & 92.3 \\
\hline & DT & $0.832(0.744-0.919)$ & 1.000 & 90.9 & 79.4 & 80.8 \\
\hline & SVM & $0.796(0.691-0.901)$ & 0.000 & 82.0 & 96.8 & 46.2 \\
\hline & Clinical & $0.845(0.757-0.934)$ & 0.000 & 82.0 & 84.1 & 76.9 \\
\hline & Combined & $0.911(0.845-0.977)$ & 0.000 & 85.4 & 87.3 & 80.8 \\
\hline \multirow[t]{5}{*}{ Validation } & LR & $0.702(0.507-0.898)$ & 0.023 & 92.9 & 50.0 & 81.3 \\
\hline & DT & $0.629(0.429-0.830)$ & 0.909 & 62.5 & 64.3 & 58.3 \\
\hline & SVM & $0.714(0.524-0.904)$ & 0.018 & 75.0 & 85.7 & 50.0 \\
\hline & Clinical & $0.786(0.638-0.933)$ & 0.002 & 77.5 & 78.6 & 75.0 \\
\hline & Combined & 0.818 (0.659-0.978) & 0.001 & 82.5 & 85.7 & 75.0 \\
\hline
\end{tabular}

$L R$, logistic regression; DT, decision tree; SVM, support vector machine; AUC, area under the receiver operating characteristic curve (ROC); Cl, confidence interval.

The correlation between radiomics features and SBRT outcomes has shown promising results. Lafata et al. investigated the relationship between pre-SBRT CT radiomics features and cancer recurrence for nonsmall cell lung cancer (NSCLC) and concluded that radiomics features may provide more predictive information in the identification of tumor local failures (37). Mattonen et al. compared the prognostic value of physician and radiomics data for local response of NSCLC treated with SBRT. Their findings similarly indicated that a radiomics score consisting of five appearance features can early and correctly predict local recurrence in a noninvasive way (18). Our results are consistent with these published studies $(18,20$, $21,37)$, as we found radiomics features were independent prognostic factors and radiomics score was significantly associated with tumor local status for primary and secondary lung cancer undergoing SBRT. These results may explain that radiomics has the ability to quantify tumor spatial and temporal heterogeneity by mathematically analyzing the spatial distribution and relationships of gray levels in CT images.

Many studies have focused on the effects of clinical and dosimetric parameters on local control $(9,10)$; however, no consensus has been reached so far and further investigation is needed. Ohri et al. developed a local TCP model with BED and tumor diameter for NSCLC patients after SBRT (14), while Ye et al. established a nomogram model with tumor size and SUVmax to predict 2-year locoregional recurrence and 2-year progression-free survival (7). However, their analysis lacked additional SBRT datasets for reliability verification and the SUVmax based on PET-CT is not easy to obtain. Our study avoided these limitations and showed that clinical stage, PLT value, and $B E D G T V_{\text {min }}$ were significantly correlated with the local control status. This finding is also in accordance with other studies (38-40). These results suggest that earlier clinical stage, lower PLT value and higher BEDGTV $\mathrm{min}_{\min }$ contribute to tumor 
A

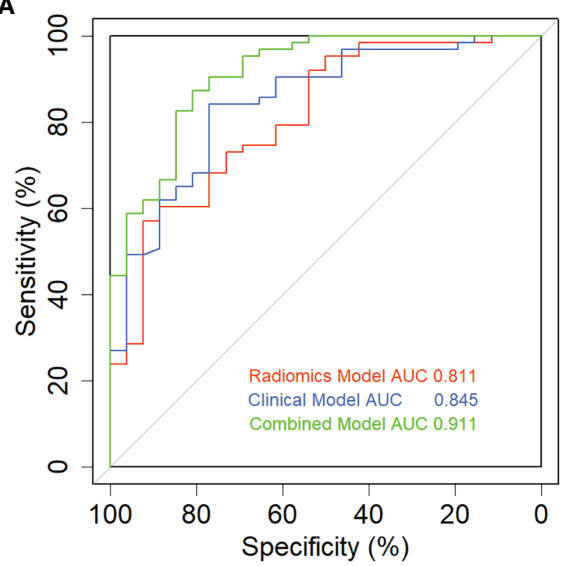

B

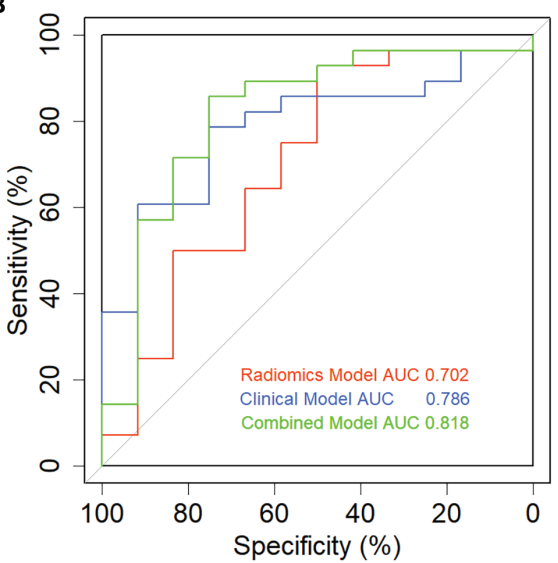

C

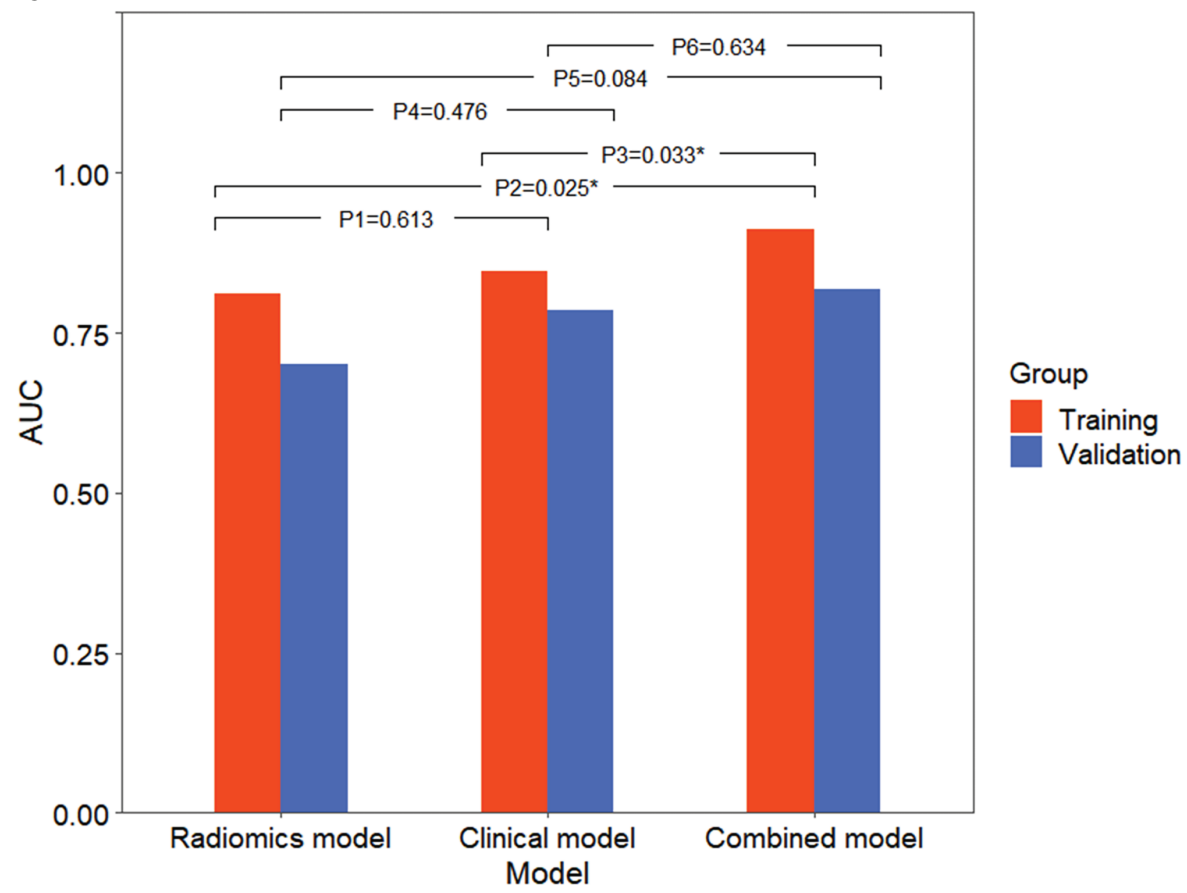

FIGURE 6 | Receiver operating characteristic (ROC) curve of three models and comparison of ROC curves. ROC curve of three models in the training group (A) and validation group (B). (C) Comparison of ROC curves with DeLong test in the training group and validation group.P1, P2, and P3 are in the training group; P4, P5, and P6 are in the validation group; P1 and P4: radiomics model vs. clinical model; P2 and P4: radiomics model vs. combined model; P3 and P6: clinical model vs. combined model. ${ }^{*} p<0.05$, expressive significance.

local control and should be considered when designing SBRT regimens.

However, these previous studies mainly focused on radiomics features $(18,20,21,37)$ or clinical and dosimetric parameters $(7$, $14,15,41)$. In our work, we combined radiomics features and the available clinical and dosimetric parameters to improve the prediction performance and accuracy for local control in lung SBRT. The combined model indicated outstanding performance in the training group and had good stability in the validation group. It failed to achieve statistical significance due to the small sample size, subtle differences in the data set or the mixed effect of other parameters. Similar results were obtained by Avanzo et al. who have demonstrated that combining BED features and image features in radiomics and deep learning improves the tumor response prediction of machine learning models for lung SBRT (42). This trend is in agreement with past studies, showing it is highly valuable to predict tumor local control in lung SBRT using multivariate factors (43). Meanwhile, the combined nomogram-integrated multiple features increases the value of personalized estimation and has great clinical application 


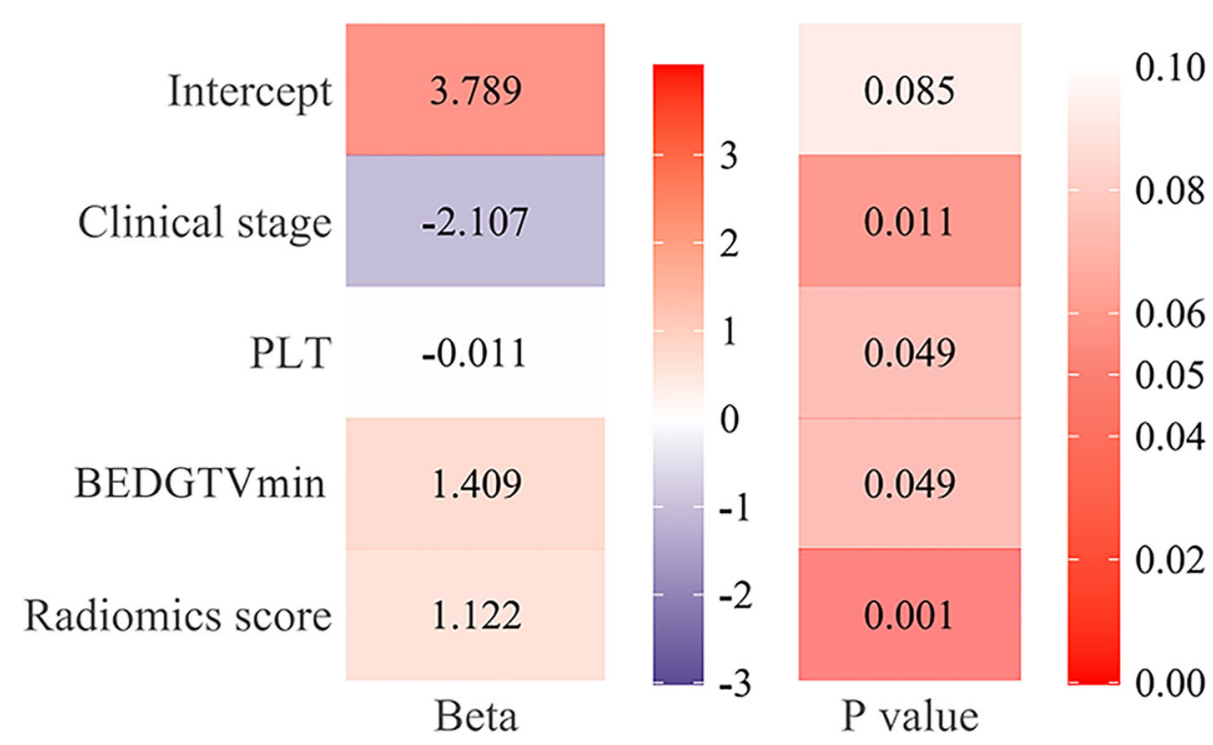

FIGURE 7 | The function of each feature in the combined model. The beta value and the $p$-value of radiomics score, clinical stage [III IV], platelet (PLT), and BEDGTV $_{\min }[\geq 98.79]$ in the combined model.

potential (19). More importantly, the variables involved in nomogram are derived from clinically available data without the need for additional expense, which will increase the clinical applicability.

It is worth noting that the incidence of local control in our study is lower than that reported previously. It might be due to the inconsistent design of treatment plans and the selection bias between different studies. According to our nomogram, the radiation dose should be increased for patients with a high risk of local recurrence. However, considering the patient's condition and nearby organs at risk, the $\mathrm{BED}_{95}$ used in our cohort, at a median of $95.2 \mathrm{~Gy}$, was lower than the standard dose of $100 \mathrm{~Gy}$ (2). In addition, some metastatic lung cancer patients were treated with significantly lower radiation doses, such as 20 35 Gy in 4 7 fractions. This was probably the reason for the high local recurrence rate in our study. In our univariate analysis, $\mathrm{BED}_{95}$ was also a significant parameter. Due to the multicollinearity between dosimetric parameters, $\mathrm{BED}_{95}$ was removed from the final model by the stepwise regression methods. However, the last dosimetric parameters entering the

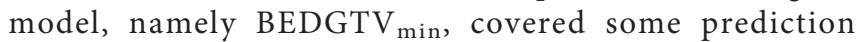
information of the $\mathrm{BED}_{95}$. Also with more data, a continued work on verifying these results is imperative.

Finally, efforts have been made to reduce the risk of radiomics feature biases and improve the quality of the prediction models. A wide range of candidate radiomics features were extracted in our study, which provided the foundation for algorithms to select relevant radiomics features and obtained the valuable information to reflect the local control status of lung cancer lesions (24). In order to reduce the deviation of the interobservers and examine the feature stability, we calculated the ICC of radiomics features (44). In addition, we optimized feature selection by using univariate analysis, LASSO, and stepwise regression, thus ensuring the independence and robustness of each feature entering the final prediction model (26). Three popular classifiers were utilized to evaluate the performance of the radiomics model, and finally the classifier with the best accuracy and the highest prognostic performance was used to establish the prediction model $(29,30)$.

There are some limitations that should be considered in the study (1): Our study was a retrospective, single-center-based study and limited number of patients were involved in the study, and results from a prospective multicenter study with a greater number of population are needed (2). Local tumor failure was not pathologically confirmed by biopsy in the study, which added more uncertainty to our conclusions. However, data from the literature show that histological confirmation was not mandatory in NSCLC patients treated with SBRT (45). (3) Our study analyzed the tumor local control status from a mixture of primary and secondary lung tumor patients. However, it was found in the previous study that TCP models were not different between primary NSCLC and secondary NSCLC, because histological heterogeneity does not influence radiosensitivity of tumor in the SBRT (15). (4) Due to the limited sample size, our endpoint mainly focused on 1-year local control and further work is required to conduct a longer follow-up time and verify the practicability of the prediction model.

\section{CONCLUSIONS}

We found that there was a significant quantitative correlation between radiomics score and local control for patients 
Points

ClinicalStage

PLT

BEDGTVmin

RadiomicsScore

Total Points

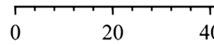

I II

III IV

$\stackrel{2}{<}$
Tumor Control Probability

B

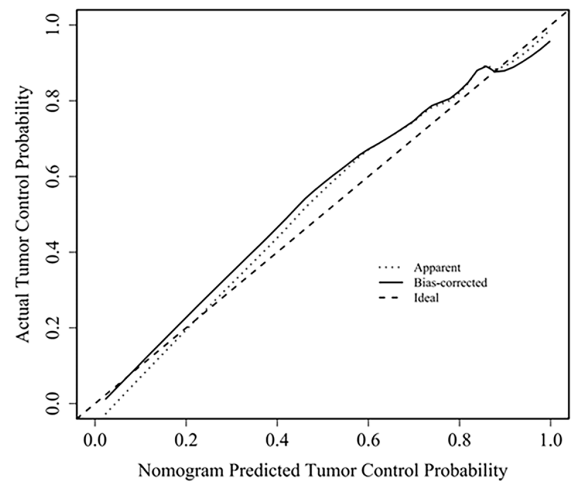

D

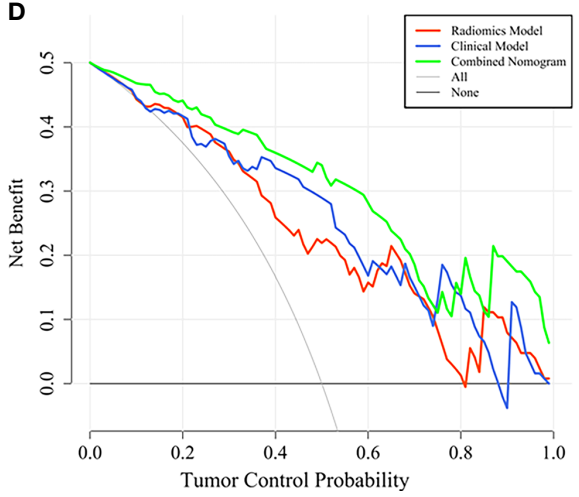

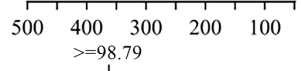

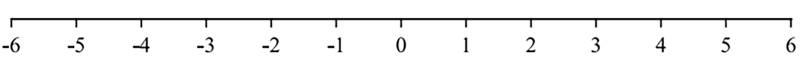

60

C

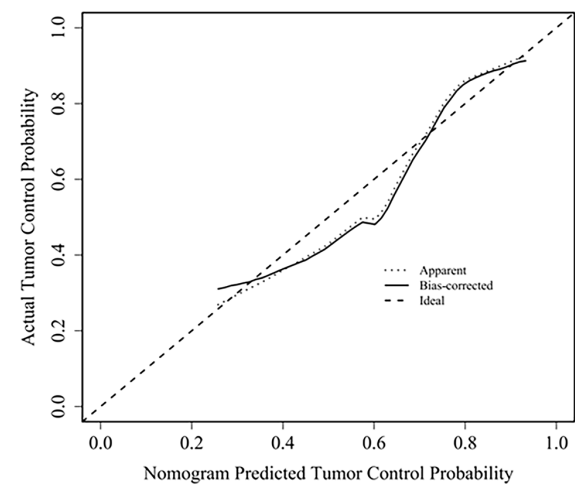

E

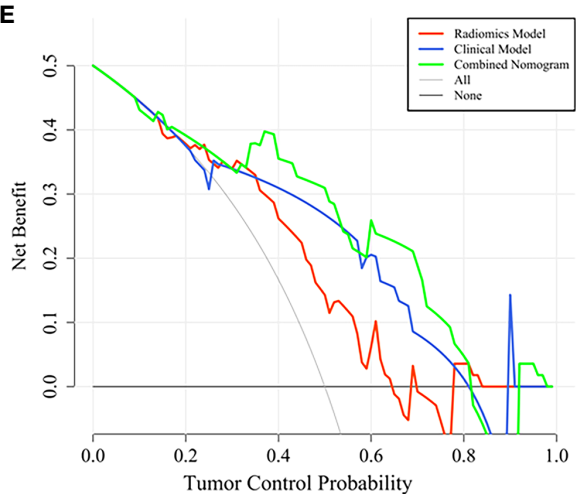

FIGURE 8 | Combined nomogram and evaluation of the nomogram in the training group and validation group. (A) A combined nomogram for prediction 1-year local control probability in lung cancer patients after SBRT. The calibration curve of the combined nomogram in the training group (B) and validation group (C). The decision curve of each model in the training group (D) and validation group (E).

undergoing SBRT, and we consider that it might be a promising and potential biomarker. According to the LR method, we developed a novel model using radiomics score plus clinical and dosimetric parameters to improve the prediction of local control. The nomogram we established have a potential to be a noninvasive, low-cost approach and could facilitate individualized treatment and follow-up, surveillance, and evaluation strategies for patients undergoing SBRT.

\section{DATA AVAILABILITY STATEMENT}

The raw data supporting the conclusions of this article will be made available by the authors, without undue reservation.

\section{AUTHOR CONTRIBUTIONS}

The author's responsibilities are as follows: B-TH, C-ZC, and L-ML conceived and designed the study. C-HS, L-ML, YW, 
R-HW, KH, and Z-HQ contributed to data collection. L-ML, G$\mathrm{BP}, \mathrm{C}-\mathrm{BZ}$, and Y-XW analyzed data and interpreted THE data. L-ML and B-TH wrote the paper. B-TH had primary responsibility for final content. All authors contributed to the manuscript review and approved the final version.

\section{FUNDING}

This work was sponsored by THE National Natural Science Foundation of China (81602667), Medical Scientific Research Foundation of Guangdong Province (A2015534, B2016048), and the Creative and Facilitating Program of Shantou University.

\section{REFERENCES}

1. Sung H, Ferlay J, Siegel RL, Laversanne M, Soerjomataram I, Jemal A, et al. Global Cancer Statistics 2020: GLOBOCAN Estimates of Incidence and Mortality Worldwide for 36 Cancers in 185 Countries. CA Cancer J Clin (2021) 71(3):209-49. doi: 10.3322/caac.21660

2. Ettinger DS, Wood DE, Aisner DL, Akerley W, Bauman J, Chirieac LR, et al. Non-Small Cell Lung Cancer, Version 5.2017, NCCN Clinical Practice Guidelines in Oncology. J Natl Compr Canc Netw (2017) 15(4):504-35. doi: 10.6004/jnccn.2017.0050

3. Rusthoven KE, Kavanagh BD, Burri SH, Chen C, Cardenes H, Chidel MA, et al. Multi-Institutional Phase I/II Trial of Stereotactic Body Radiation Therapy for Lung Metastases. J Clin Oncol (2009) 27(10):1579-84. doi: $10.1200 /$ jco.2008.19.6386

4. Schneider BJ, Daly ME, Kennedy EB, Antonoff MB, Broderick S, Feldman J, et al. Stereotactic Body Radiotherapy for Early-Stage Non-Small-Cell Lung Cancer: American Society of Clinical Oncology Endorsement of the American Society for Radiation Oncology Evidence-Based Guideline. J Clin Oncol (2018) 36(7):710-9. doi: 10.1200/jco.2017.74.9671

5. Timmerman R, Paulus R, Galvin J, Michalski J, Straube W, Bradley J, et al. Stereotactic Body Radiation Therapy for Inoperable Early Stage Lung Cancer. JAMA (2010) 303(11):1070-6. doi: 10.1001/jama.2010.261

6. Takeda A, Sanuki N, Fujii H, Yokosuka N, Nishimura S, Aoki Y, et al. Maximum Standardized Uptake Value on FDG-PET Is a Strong Predictor of Overall and Disease-Free Survival for Non-Small-Cell Lung Cancer Patients After Stereotactic Body Radiotherapy. J Thorac Oncol (2014) 9(1):65-73. doi: 10.1097/jto.0000000000000031

7. Ye L, Shi S, Zeng Z, Huang Y, Hu Y, He J. Nomograms for Predicting Disease Progression in Patients of Stage I Non-Small Cell Lung Cancer Treated With Stereotactic Body Radiotherapy. Jpn J Clin Oncol (2018) 48(2):160-6. doi: 10.1093/jjco/hyx179

8. Hörner-Rieber J, Bernhardt D, Dern J, König L, Adeberg S, Paul A, et al. Histology of Non-Small Cell Lung Cancer Predicts the Response to Stereotactic Body Radiotherapy. Radiother Oncol (2017) 125(2):317-24. doi: 10.1016/j.radonc.2017.08.029

9. Woody NM, Stephans KL, Andrews M, Zhuang T, Gopal P, Xia P, et al. A Histologic Basis for the Efficacy of SBRT to the Lung. J Thorac Oncol (2017) 12(3):510-9. doi: 10.1016/j.jtho.2016.11.002

10. Kestin L, Grills I, Guckenberger M, Belderbos J, Hope AJ, Werner-Wasik M, et al. Dose-Response Relationship With Clinical Outcome for Lung Stereotactic Body Radiotherapy (SBRT) Delivered via Online Image Guidance. Radiother Oncol (2014) 110(3):499-504. doi: 10.1016/ j.radonc.2014.02.002

11. Diamant A, Chatterjee A, Faria S, Naqa IE, Bahig H, Filion E, et al. Can Dose Outside the PTV Influence the Risk of Distant Metastases in Stage I Lung Cancer Patients Treated With Stereotactic Body Radiotherapy (SBRT)? Radiother Oncol (2018) 128(3):513-9. doi: 10.1016/j.radonc.2018.05.012

12. Guckenberger M, Klement RJ, Allgäuer M, Appold S, Dieckmann K, Ernst I, et al. Applicability of the Linear-Quadratic Formalism for Modeling Local Tumor Control Probability in High Dose Per Fraction Stereotactic Body

\section{ACKNOWLEDGMENTS}

We thank all participants and staff for their endeavor and contribution in this study. We thank our hospital for their support of this research project.

\section{SUPPLEMENTARY MATERIAL}

The Supplementary Material for this article can be found online at: https://www.frontiersin.org/articles/10.3389/fonc.2021.819047/ full\#supplementary-material

Radiotherapy for Early Stage Non-Small Cell Lung Cancer. Radiother Oncol (2013) 109(1):13-20. doi: 10.1016/j.radonc.2013.09.005

13. Mehta N, King CR, Agazaryan N, Steinberg M, Hua A, Lee P. Stereotactic Body Radiation Therapy and 3-Dimensional Conformal Radiotherapy for Stage I Non-Small Cell Lung Cancer: A Pooled Analysis of Biological Equivalent Dose and Local Control. Pract Radiat Oncol (2012) 2(4):288-95. doi: 10.1016/j.prro.2011.10.004

14. Ohri N, Werner-Wasik M, Grills IS, Belderbos J, Hope A, Yan D, et al. Modeling Local Control After Hypofractionated Stereotactic Body Radiation Therapy for Stage I Non-Small Cell Lung Cancer: A Report From the Elekta Collaborative Lung Research Group. Int J Radiat Oncol Biol Phys (2012) 84(3): e379-84. doi: 10.1016/j.ijrobp.2012.04.040

15. Guckenberger M, Klement RJ, Allgäuer M, Andratschke N, Blanck O, BodaHeggemann J, et al. Local Tumor Control Probability Modeling of Primary and Secondary Lung Tumors in Stereotactic Body Radiotherapy. Radiother Oncol (2016) 118(3):485-91. doi: 10.1016/j.radonc.2015.09.008

16. Lambin P, Leijenaar RTH, Deist TM, Peerlings J, de Jong EEC, van Timmeren J, et al. Radiomics: The Bridge Between Medical Imaging and Personalized Medicine. Nat Rev Clin Oncol (2017) 14(12):749-62. doi: 10.1038/nrclinonc. 2017.141

17. Aerts HJ, Velazquez ER, Leijenaar RT, Parmar C, Grossmann P, Carvalho S, et al. Decoding Tumour Phenotype by Noninvasive Imaging Using a Quantitative Radiomics Approach. Nat Commun (2014) 5:4006. doi: 10.1038/ncomms5006

18. Mattonen SA, Palma DA, Johnson C, Louie AV, Landis M, Rodrigues G, et al. Detection of Local Cancer Recurrence After Stereotactic Ablative Radiation Therapy for Lung Cancer: Physician Performance Versus Radiomic Assessment. Int J Radiat Oncol Biol Phys (2016) 94(5):1121-8. doi: 10.1016/ j.ijrobp.2015.12.369

19. Huang Y, Liu Z, He L, Chen X, Pan D, Ma Z, et al. Radiomics Signature: A Potential Biomarker for the Prediction of Disease-Free Survival in Early-Stage (I or II) Non-Small Cell Lung Cancer. Radiology (2016) 281(3):947-57. doi: 10.1148/radiol.2016152234

20. Huynh E, Coroller TP, Narayan V, Agrawal V, Hou Y, Romano J, et al. CTBased Radiomic Analysis of Stereotactic Body Radiation Therapy Patients With Lung Cancer. Radiother Oncol (2016) 120(2):258-66. doi: 10.1016/ j.radonc.2016.05.024

21. Yu W, Tang C, Hobbs BP, Li X, Koay EJ, Wistuba II, et al. Development and Validation of a Predictive Radiomics Model for Clinical Outcomes in Stage I Non-Small Cell Lung Cancer. Int J Radiat Oncol Biol Phys (2018) 102 (4):1090-7. doi: 10.1016/j.ijrobp.2017.10.046

22. Lenglet A, Campeau MP, Mathieu D, Bahig H, Lambert L, Vu T, et al. RiskAdapted Stereotactic Ablative Radiotherapy for Central and Ultra-Central Lung Tumours. Radiother Oncol (2019) 134:178-84. doi: 10.1016/j.radonc. 2019.01.035

23. Zhang L, Chen B, Liu X, Song J, Fang M, Hu C, et al. Quantitative Biomarkers for Prediction of Epidermal Growth Factor Receptor Mutation in Non-Small Cell Lung Cancer. Transl Oncol (2018) 11(1):94-101. doi: 10.1016/j.tranon. 2017.10.012

24. Tao J, Lv R, Liang C, Fang J, Liu D, Lan X, et al. Development and Validation of a CT-Based Signature for the Prediction of Distant Metastasis Before 
Treatment of Non-Small Cell Lung Cancer. Acad Radiol (2021) S1076-6332 (20):30700-5. doi: 10.1016/j.acra.2020.12.007

25. Kim SB, Heo YM, Hwang CM, Kim TG, Hong JY, Won YG, et al. Reliability of the EOS Imaging System for Assessment of the Spinal and Pelvic Alignment in the Sagittal Plane. Clin Orthop Surg (2018) 10(4):500-7. doi: 10.4055/ cios.2018.10.4.500

26. Whittingham MJ, Stephens PA, Bradbury RB, Freckleton RP. Why Do We Still Use Stepwise Modelling in Ecology and Behaviour? J Anim Ecol (2006) 75 (5):1182-9. doi: 10.1111/j.1365-2656.2006.01141.x

27. Tibshirani R. Regression Shrinkage and Selection via the Lasso: A Retrospective. J R Stat Soc B (2011) 73(3):267-88. doi: 10.1111/j.14679868.2011.00771.x

28. Chen Y, Chen TW, Wu CQ, Lin Q, Hu R, Xie CL, et al. Radiomics Model of Contrast-Enhanced Computed Tomography for Predicting the Recurrence of Acute Pancreatitis. Eur Radiol (2019) 29(8):4408-17. doi: 10.1007/s00330018-5824-1

29. Bektas CT, Kocak B, Yardimci AH, Turkcanoglu MH, Yucetas U, Koca SB, et al. Clear Cell Renal Cell Carcinoma: Machine Learning-Based Quantitative Computed Tomography Texture Analysis for Prediction of Fuhrman Nuclear Grade. Eur Radiol (2019) 29(3):1153-63. doi: 10.1007/s00330-018-5698-2

30. Zhou Y, Ma XL, Zhang T, Wang J, Zhang T, Tian R. Use of Radiomics Based on (18)F-FDG PET/CT and Machine Learning Methods to Aid Clinical Decision-Making in the Classification of Solitary Pulmonary Lesions: An Innovative Approach. Eur J Nucl Med Mol Imaging (2021) 48(9):2904-13. doi: 10.1007/s00259-021-05220-7

31. He B, Song Y, Wang L, Wang T, She Y, Hou L, et al. A Machine LearningBased Prediction of the Micropapillary/Solid Growth Pattern in Invasive Lung Adenocarcinoma With Radiomics. Transl Lung Cancer Res (2021) 10(2):95564. doi: $10.21037 /$ tlcr-21-44

32. Rau CS, Wu SC, Chien PC, Kuo PJ, Chen YC, Hsieh HY, et al. Identification of Pancreatic Injury in Patients With Elevated Amylase or Lipase Level Using a Decision Tree Classifier: A Cross-Sectional Retrospective Analysis in a Level I Trauma Center. Int J Environ Res Public Health (2018) 15(2):277. doi: 10.3390/ijerph15020277

33. Chen H, Zeng M, Wang X, Su L, Xia Y, Yang Q, et al. A CT-Based Radiomics Nomogram for Predicting Prognosis of Coronavirus Disease 2019 (COVID19) Radiomics Nomogram Predicting COVID-19. Br J Radiol (2021) 94 (1117):20200634. doi: 10.1259/bjr.20200634

34. Douma RA, le Gal G, Söhne M, Righini M, Kamphuisen PW, Perrier A, et al. Potential of an Age Adjusted D-Dimer Cut-Off Value to Improve the Exclusion of Pulmonary Embolism in Older Patients: A Retrospective Analysis of Three Large Cohorts. Bmj (2010) 340:c1475. doi: 10.1136/ bmj.c1475

35. Theelen W, Chen D, Verma V, Hobbs BP, Peulen HMU, Aerts J, et al. Pembrolizumab With or Without Radiotherapy for Metastatic Non-SmallCell Lung Cancer: A Pooled Analysis of Two Randomised Trials. Lancet Respir Med (2021) 9(5):467-75. doi: 10.1016/s2213-2600(20)30391-x

36. Tian S, Switchenko JM, Buchwald ZS, Patel PR, Shelton JW, Kahn SE, et al. Lung Stereotactic Body Radiation Therapy and Concurrent Immunotherapy: A Multicenter Safety and Toxicity Analysis. Int J Radiat Oncol Biol Phys (2020) 108(1):304-13. doi: 10.1016/j.ijrobp.2019.12.030

37. Lafata KJ, Hong JC, Geng R, Ackerson BG, Liu JG, Zhou Z, et al. Association of Pre-Treatment Radiomic Features With Lung Cancer Recurrence
Following Stereotactic Body Radiation Therapy. Phys Med Biol (2019) 64 (2):025007. doi: 10.1088/1361-6560/aaf5a5

38. Ernani V, Appiah AK, Marr A, Zhang C, Zhen W, Smith LM, et al. Adjuvant Systemic Therapy in Patients With Early-Stage NSCLC Treated With Stereotactic Body Radiation Therapy. J Thorac Oncol (2019) 14(3):475-81. doi: 10.1016/j.jtho.2018.11.018

39. Dunlap NE, Larner JM, Read PW, Kozower BD, Lau CL, Sheng K, et al. Size Matters: A Comparison of T1 and T2 Peripheral Non-Small-Cell Lung Cancers Treated With Stereotactic Body Radiation Therapy (SBRT). J Thorac Cardiovasc Surg (2010) 140(3):583-9. doi: 10.1016/j.jtcvs.2010.01.046

40. Inoue H, Kosuga T, Kubota T, Konishi H, Shiozaki A, Okamoto K, et al. Significance of a Preoperative Systemic Immune-Inflammation Index as a Predictor of Postoperative Survival Outcomes in Gastric Cancer. World J Surg Oncol (2021) 19(1):173. doi: 10.1186/s12957-021-02286-3

41. Klement RJ, Allgäuer M, Andratschke N, Blanck O, Boda-Heggemann J, Dieckmann K, et al. Bayesian Cure Rate Modeling of Local Tumor Control: Evaluation in Stereotactic Body Radiation Therapy for Pulmonary Metastases. Int J Radiat Oncol Biol Phys (2016) 94(4):841-9. doi: 10.1016/ j.ijrobp.2015.12.004

42. Avanzo M, Gagliardi V, Stancanello J, Blanck O, Pirrone G, El Naqa I, et al. Combining Computed Tomography and Biologically Effective Dose in Radiomics and Deep Learning Improves Prediction of Tumor Response to Robotic Lung Stereotactic Body Radiation Therapy. Med Phys (2021) 48 (10):6257-69. doi: 10.1002/mp.15178

43. Grossmann P, Stringfield O, El-Hachem N, Bui MM, Rios Velazquez E, Parmar C, et al. Defining the Biological Basis of Radiomic Phenotypes in Lung Cancer. Elife (2017) 6:23421. doi: 10.7554/eLife.23421

44. Wang L, Gao Z, Li C, Sun L, Li J, Yu J, et al. Computed Tomography-Based Delta-Radiomics Analysis for Discriminating Radiation Pneumonitis in Patients With Esophageal Cancer After Radiation Therapy. Int J Radiat Oncol Biol Phys (2021) 111(2):443-55. doi: 10.1016/j.ijrobp.2021.04.047

45. Videtic GMM, Donington J, Giuliani M, Heinzerling J, Karas TZ, Kelsey CR, et al. Stereotactic Body Radiation Therapy for Early-Stage Non-Small Cell Lung Cancer: Executive Summary of an ASTRO Evidence-Based Guideline. Pract Radiat Oncol (2017) 7(5):295-301. doi: 10.1016/j.prro.2017.04.014

Conflict of Interest: The authors declare that the research was conducted in the absence of any commercial or financial relationships that could be construed as a potential conflict of interest.

Publisher's Note: All claims expressed in this article are solely those of the authors and do not necessarily represent those of their affiliated organizations, or those of the publisher, the editors and the reviewers. Any product that may be evaluated in this article, or claim that may be made by its manufacturer, is not guaranteed or endorsed by the publisher.

Copyright (๐ 2022 Luo, Huang, Chen, Wang, Su, Peng, Zeng, Wu, Wang, Huang and Qiu. This is an open-access article distributed under the terms of the Creative Commons Attribution License (CC BY). The use, distribution or reproduction in other forums is permitted, provided the original author(s) and the copyright owner(s) are credited and that the original publication in this journal is cited, in accordance with accepted academic practice. No use, distribution or reproduction is permitted which does not comply with these terms. 


\section{GLOSSARY}

\begin{tabular}{|c|c|}
\hline SBRT & stereotactic body radiation therapy \\
\hline CT & Computed tomography \\
\hline SUVmax & maximum standardized uptake value \\
\hline 4DCT & four-dimensional computed tomography \\
\hline 3DCT & three-dimensional computed tomography \\
\hline CBCT & Cone beam computed tomography \\
\hline GTV & gross tumor volume \\
\hline ITV & internal target volume \\
\hline PTV & planning target volume \\
\hline BED & biologically effective dose \\
\hline KPS & Karnofsky performance status \\
\hline BMl & body mass index \\
\hline PLT & platelet \\
\hline $\mathrm{Hb}$ & hemoglobin \\
\hline NLR & neutrophil-to-lymphocyte ratio \\
\hline PLR & platelet-to-lymphocyte ratio \\
\hline $\mathrm{BED}_{95}$ & $\begin{array}{l}\text { the prescription dose covers } 95 \% \text { of the target area } \\
\text { expressed as BED }\end{array}$ \\
\hline $\mathrm{BED}_{\max }$ & the maximum dose in the whole plan \\
\hline BEDPTV $_{\min }$ & the minimum dose of PTV \\
\hline $\mathrm{BEDPTV}_{\text {mean }}$ & mean dose of PTV \\
\hline BEDPTV $_{\max }$ & the maximum dose of PTV \\
\hline $\begin{array}{l}\text { BEDPTV }_{\min } / \\
\text { PTV }_{\max }\end{array}$ & dose inhomogeneity in PTV \\
\hline BEDGTV $_{\min }$ & the minimum dose of GTV \\
\hline BEDGTV $V_{\text {mean }}$ & mean dose of GTV \\
\hline BEDGTV $_{\max }$ & the maximum dose of GTV \\
\hline $\begin{array}{l}\text { BEDGTV }_{\min } / \\
\mathrm{GTV}_{\max }\end{array}$ & dose inhomogeneity in GTV \\
\hline $\mathrm{ROI}$ & region of interest \\
\hline GLCM & gray-level co-occurrence matrix \\
\hline GLDM & gray-level dependence matrix \\
\hline GLRLM & gray-level run length matrix \\
\hline GLSZM & gray-level size zone matrix \\
\hline NGTDM & neighborhood gray-tone difference matrix \\
\hline ICC & intraclass correlation coefficient \\
\hline LASSO & least absolute shrinkage and selection operator \\
\hline LR & logistic regression \\
\hline DT & decision tree \\
\hline SVM & support vector machine \\
\hline $\mathrm{ROC}$ & receiver operating characteristic \\
\hline AUC & area under the ROC curve \\
\hline $\mathrm{Cl}$ & confidence interval \\
\hline NSCLC & nonsmall cell lung cancer \\
\hline TCP & tumor control probability \\
\hline LVI & lymphovascular invasion \\
\hline PET-CT & positron emission tomography-CT \\
\hline
\end{tabular}

